\title{
Le manuscrit retrouvé des Mémoires de Nicolas
}

\section{Fontaine}

ou Mémoire pour servir à l'histoire de l'entretien de Pascal avec M. de Sacy

Pascale Mengotti-Thouvenin

\section{(2) OpenEdition}

\section{Journals}

Édition électronique

URL : https://journals.openedition.org/ccibp/582

DOI : $10.4000 /$ ccibp.582

ISSN : 2493-7460

Éditeur

Centre international Blaise Pascal

Édition imprimée

Date de publication : 10 décembre 1995

Pagination : 32-36

ISSN : 0249-6674

Référence électronique

Pascale Mengotti-Thouvenin, «Le manuscrit retrouvé des Mémoires de Nicolas Fontaine », Courrier du Centre International Blaise-Pascal [En ligne], 17 | 1995, mis en ligne le 07 janvier 2016, consulté le 28 juin 2022. URL : http://journals.openedition.org/ccibp/582 ; DOI : https://doi.org/10.4000/ccibp.582

Ce document a été généré automatiquement le 29 septembre 2020

Centre international Blaise Pascal 


\title{
Le manuscrit retrouvé des Mémoires de Nicolas Fontaine
}

ou Mémoire pour servir à l'histoire de l'entretien de Pascal avec M. de Sacy

\author{
Pascale Mengotti-Thouvenin
}

\section{Les étapes d'une improbable découverte}

1 Comment et où découvrir un inédit, et de surcroît un original autographe ? La réponse déconcertera sans doute: sans le chercher et dans le fonds d'une bibliothèque accessible à tous. On ne dira jamais assez quelles surprises peuvent surgir de l'exploration tâtonnante, mais non sans but, des catalogues des manuscrits, défendus dans les bibliothèques publiques par l'altitude maligne et les secrètes profondeurs des rayonnages, sous l'indiscrète poussière et dans un éclairage en général parcimonieux. Ainsi, offert à tous mais mystérieusement ignoré, se tenait dûment répertorié dans le catalogue des manuscrits de la bibliothèque de l'Institut de France, et selon tous les principes de la bibliographie, un manuscrit des Mémoires de Nicolas Fontaine qui ne figurait dans aucune recension ${ }^{1}$. Et qui par conséquent présentait un texte inédit du célèbre Entretien de Pascal avec M. de Sacy.

2 L'examen de l'Entretien révélait un texte entièrement nouveau, où se trouvaient résolues comme par enchantement les énigmes qu'une critique éminente s'était efforcée de résoudre ${ }^{2}$. On ne pouvait hésiter à attribuer à ce nouveau manuscrit un rang qui surclassait tous les autres connus. Mais était-il possible que l'improbable se fût produit, que l'original considéré comme perdu depuis des générations fût retrouvé ? On formulait les hypothèses incertaines d'un débutant, il fallait l'autorité impeccable d'un maître. Jean Mesnard se prononçait : le manuscrit nouveau était l'original autographe retrouvé, donné pour introuvable ${ }^{3}$.

Comment était-on arrivé à ce résultat? On se proposait d'éditer les Mémoires de Nicolas Fontaine. Publiés en 1736 par les soins de Michel Tronchay ${ }^{4}$, ancien secrétaire de Sébastien Le Nain de Tillemont et dirigé de Godefroy Hermant, les Mémoires avaient 
paru fortement abrégés. Tous les historiens cependant leur reconnaissaient un intérêt de premier plan ${ }^{5}$. Entré à l'âge de vingt ans à Port-Royal en 1644, Nicolas Fontaine avait lié sa destinée à celle de Port-Royal et de ses membres jusqu'à sa mort en 1709. Attaché en qualité de secrétaire d'abord aux Messieurs, puis particulièrement à Isaac Le Maistre de Sacy, maître aux Petites Écoles, le solitaire avait connu l'emprisonnement avec Sacy à la Bastille, de 1666 à 1668, et tous les événements religieux, théologiques, politiques, littéraires qui avaient intéressé Port-Royal ${ }^{6}$. Ses Mémoires, composés dans la retraite de ses dernières années, de 1696 à 1700, n'étaient cependant pas accessibles autrement que dans cette unique édition, ancienne, réalisée d'après un manuscrit lacunaire et très fautif, éloigné de la source. Un travail de restitution du texte original s'imposait, auquel l'extraordinaire développement, depuis les trente dernières années, des études sur Port-Royal d'une part, et sur le genre des mémoires d'autre part, était propice.

Une lettre de Michel Tronchay venait ajouter une motivation nouvelle. Celui-ci en effet présentait en ces termes son projet d'édition des Mémoires :

On m'a envoyé à revoir l'Histoire des Solitaires de Port-Royal par M. Fontaine que j'ai connu. Ce n'est rien moins qu'une histoire qui n'a ni ordre, ni chronologie, ni narration suivie. Ce sont des épanchements du cœur de ce bonhomme. On en peut retrancher la moitié sans en rien ôter d'intéressant. En un mot, c'est un lambeau de ces Vies de Saints, farci de réflexions ennuyeuses et de prières répétées jusqu'à la nausée. J'en change le titre...j'abrègerai toutes ses réflexions, et j'en ôterai entièrement quelques-unes... ${ }^{7}$ "

5 En donnant les raisons de ses choix, en restreignant le texte de Fontaine pour le resserrer dans les bornes de la sensibilité et des attentes d'un lecteur du début du dixhuitième siècle, Tronchay ouvrait de nouvelles perspectives à l'éditeur moderne, laissait entrevoir des explorations inédites, des découvertes inattendues. On pressentait, sous les réticences à évoquer les "épanchements du cœur", le "lambeau des Vies de Saints", les "réflexions ennuyeuses", un véritable objet littéraire, défiguré, mutilé, qui attendait depuis près de trois siècles qu'on lui rendît son visage authentique et qu'on fît entendre sa véritable voix. Un être exigeait d'outre-tombe qu'on lui rendît justice.

\section{Les énigmes d'un manuscrit en attente d'impression}

Mais le manuscrit réservait une autre surprise. En effet, dans les «nombreuses additions et annotations " évoquées dans le catalogue ${ }^{8}$, on découvrait de nombreux documents témoignant d'un projet d'édition. Muni d'une préface, d'un avertissement, de corrections et de traductions du latin, le manuscrit a été l'objet d'un travail d'édition organisé dont on ne peut mettre en doute la continuité. L'exécution du projet s'est trouvée interrompue à un stade très poussé des étapes de la publication. En effet certaines remarques sont adressées directement à l'imprimeur : détails distinguant le système matériel de correction, biffures au charbon et biffures à l'encre, et même consignes pratiques pour l'impression du latin et de sa traduction. Ainsi, nous saisissons le manuscrit au moment décisif où il est confié à l'imprimeur pour la phase ultime. Il faut donc parler désormais, plutôt que d'un projet d'édition, d'une édition achevée, munie de tout ce qui avait été jugé nécessaire pour la parution des Mémoires, et à laquelle seule l'impression a manqué.

7 Pour quelle raison cette édition n'a-t-elle jamais vu le jour? C'est une nouvelle énigme qu'aucune mention de nom ni de date, dans l'abondant dossier formé par les pièces qui 
accompagnent le manuscrit, ne vient aider à résoudre. Tout au plus peut-on délimiter la période pendant laquelle l'édition a été préparée, après 1723 et avant 1759 , confirmée par le caractère ancien des écritures de l'auteur de la préface, du correcteur, et de plusieurs autres, que l'on peut expertiser comme celles de personnes du début du XVIII siècle.

Qu'en est-il, dans ces conditions, de l'édition de l'Entretien? En effet, les corrections effectuées dans l'ensemble des Mémoires vont de la modification de passages plus ou moins longs à la suppression pure et simple, dictées dans la plupart des cas soit par souci stylistique, soit par désir d'expurger un texte qui pourrait nuire à la mémoire des Messieurs. L'Entretien échappe-t-il à ces intentions? Alors que le correcteur pratique généreusement corrections et suppressions dans l'ensemble des Mémoires, très souvent il est vrai dans les très fréquents épanchements lyriques de Fontaine, l'Entretien a dans son ensemble subi un traitement qui associe plusieurs procédés. Seul un long passage est l'objet d'une suppression radicale, et encore s'agit-il d'un développement qui, dans la bouche de Sacy, prélude à sa rencontre et à son dialogue avec Pascal lors de la retraite de celui-ci à Port-Royal des Champs. En effet l'auteur inconnu d'un « Avis sur la correction de ce manuscrit ${ }^{9}$ ", qui, comme l'indique ce titre, expose les principes qui ont dirigé le travail de correction, précise: "on a passé des lignes sur certains endroits du manuscrit qui ne les empêchent pas d'être lus, mais il faut les retrancher parce qu'ils renferment des pensées et des discours dont on abuserait pour décrier les grands personnages dont il y est question. Des amis qui s'entretiennent à cœur ouvert se disent bien des choses qu'ils seraient fâchés de faire en public.» Or un passage relatif aux débats intellectuels parmi les solitaires à propos des théories cartésiennes est biffé à l'encre, ce qui indique sa suppression dans l'édition ${ }^{10}$. La volonté d'atténuer la violence des oppositions entre cartésiens et anticartésiens parmi les solitaires paraît évidente. À Sacy, dépouillé de son ironie, est attribué un comportement plus convenable. La suite du développement est l'objet d'un autre type de correction: de larges croix au charbon barrent deux pages entières ${ }^{11}$. Or, indique l'auteur de l'«Avis » ${ }^{12}$, «Ce qu'on a cru devoir retrancher comme inutile et superflu est croisé et barré avec du charbon seulement ». Cette indication est loin de donner pleinement satisfaction, car comment l'interpréter, quel sens donner à « inutile et superflu»? Quelles modalités attacher à «Ce qu'on a cru devoir... »? Y voir le désir d'abréger des développements que l'on jugerait exagérément longs ou redondants est probablement insuffisant. On note en effet, comme dans le développement précédent, une grande vigueur dans les propos de Sacy, redoutable satiriste. Or, nombreux sont, dans l'ensemble des Mémoires, les passages où le texte est corrigé de manière à redonner au solitaire "cette douceur qui lui était si naturelle ${ }^{13}$ ». Il est vraisemblable que l'intention du correcteur a été de retoucher le personnage de Sacy dans un sens plus conforme à la légende. Mais, comme semble le témoigner la traduction en français des citations latines dans les interlignes, le passage a peut-être finalement été destiné à être imprimé. Quoiqu'il en soit, la suppression de ce dialogue entre Sacy et Descartes, pour nous très précieux témoignage de la vivacité des débats à Port-Royal entre les cartésiens et leurs opposants représentés dans l'œuvre de Fontaine par la haute figure de Sacy, aurait constitué une irremplaçable perte.

9 Une fois prise la mesure de ces modifications apportées au texte, on s'étonnera davantage des rares transformations portant sur la suite de l'Entretien, à partir de l'entrée en scène de Pascal. En effet, une seule suppression notable modifie le sens du texte. Il s'agit d'un point de la doctrine d'Épictète ${ }^{14}$, dans la bouche de Pascal. Bien que présentée par celui-ci et sans aucune ambiguïté comme une « erreur », l'idée du suicide, 
intolérable pour une mentalité chrétienne, fut biffée à l'encre, et ne devait pas paraître dans l'édition imprimée. Pour le reste, il s'agit de quelques modifications qui peuvent se réduire à des corrections de style ${ }^{15}$ et qui ne transforment ni le sens ni la portée du propos. Le correcteur intervient encore en divers endroits pour donner des traductions des citations latines qui ne sont pas paraphrasées par les propos en français de Pascal ou de Sacy. Ainsi, le correcteur a porté aux échanges des deux interlocuteurs un respect étonnant dans le contexte fortement retouché de l'ensemble de l'œuvre de Fontaine. On espère y saisir la marque d'un esprit attentif et sensible qui a vu briller, au centre des Mémoires, la profonde beauté et le puissant éclat de l'Entretien. Ainsi, si l'édition préparée avait abouti à une publication, un texte presque intact aurait été offert au public dans la première moitié du XVIII ${ }^{\mathrm{e}}$ siècle, surclassant l'édition qu'en avait donné le Père Desmolets dans sa Continuation des Mémoires de littérature et d'histoire ${ }^{16}$ en 1728 , et celle de Tronchay dans sa publication des Mémoires en 1736.

\section{Des rencontres manquées}

Curieusement la suite de l'histoire du manuscrit fut celle de rencontres manquées. Évoquons l'une d'entre elle, qui n'est pas la moins surprenante : celle de l'oratorien Pierre Guerrier, cousin de Marguerite Périer, nièce de Pascal, la petite miraculée de la Sainte Épine.

Une partie de l'histoire du manuscrit des Mémoires de Fontaine nous est connue, celle où, faisant partie de la collection de manuscrits du parlementaire Antoine Moriau (1699-1759), il fut intégré au fonds de la Bibliothèque de la Ville de Paris constitué précisément par le legs que le parlementaire avait destiné à cet usage ${ }^{17}$. Le 2 septembre 1760, le Bureau de la Ville nomma pour bibliothécaire de la nouvelle bibliothèque créée grâce aux dispositions testamentaires d'Antoine Moriau, Pierre-Nicolas Bonamy (1694-1770), membre de l'Académie des Inscriptions et Belles-Lettres, historiographe du roi, qui avait précédemment fait carrière comme bibliothécaire du monastère bénédictin Saint-Victor, puis du parlementaire Le Peletier des Forts ${ }^{18}$. Or, dans une lettre du 19 juin $1760^{19}$, Bonamy remercie Charles Le Peletier de Saint-Fargeau des démarches qu'il a entreprises pour lui adjoindre dans sa tâche son ancien ami le Père Pierre Guerrier :

...Le Père Général de l'Oratoire a dit à une personne que vous lui aviez demandé de faire venir ici le P. Guerrier, et qu'il vous avait accordé votre demande. Cette facilité d'un homme qui est tout aussi méticuleux qu'un autre, et qui a toujours évité ce qui pouvait lui causer le moindre embarras, doit vous rassurer, Monsieur, sur la résidence de mon ami à Paris. Je puis vous répondre de la sagesse et de la prudence qui ont guidé jusqu'à présent sa conduite; son caractère pacifique a toujours été de se mettre à l'écart, de ne se mêler de rien, et de fuir avec attention d'être à portée de gens qui le feraient parler mal à propos ; aussi ne s'est-il jamais attiré d'affaire dans tous les endroits où il a demeuré. Enfin, si l'on pouvait louer ces terribles jansénistes, je dirais que c'est le plus aimable de tous ceux que j'ai connus. J'espère qu'avec l'ample provision de connaissances qu'il a acquise, sa présence ne vous sera pas inutile; hors la messe et les exercices de sa maison, il n'aura d'autre occupation que celle de lire et d'étudier, ainsi vous l'aurez toujours sous votre main dans ces occasions. Je dois, Monsieur mettre au nombre de tant d'obligations que je vous ai, la bonté que vous avez eue de faire revenir ici un ami auquel depuis 45 ans je suis tendrement attaché... 
On est particulièrement sensible, dans ce portrait destiné à attirer la bienveillance de Le Peletier de Saint-Fargeau, aux détails évoquant le jansénisme désormais inoffensif de l'oratorien ${ }^{20}$. On imagine sans peine quel profit eût tiré le Père Guerrier d'un labeur voué à inventorier et explorer un fonds nouvellement créé, quel champ eût été donné à "l'ample provision de connaissances qu'il a < vait > acquise ", dans un loisir où il n'aurait eu "d'autre occupation que celle de lire et d'étudier». En effet, son rôle capital dans la conservation et la transmission de l'héritage pascalien, notamment la fameuse Seconde copie des Pensées, est bien connu ${ }^{21}$. On sait en effet que l'oratorien accomplit un travail de collection d'écrits de Pascal ou concernant Pascal le plus complet possible en constituant d'immenses recueils de copies ou d'originaux dont il puisait la matière dans le fonds de la bibliothèque des oratoriens de Clermont, héritiers d'une partie des papiers de Pascal, et auprès de Marguerite Périer. Ce sont les célèbres Recueils Guerrier, constitués entre 1731 et 1736. Le Père Guerrier paraît constamment animé par le souci de faire entrer dans sa collection tout document ou souvenir concernant son célèbre parent ${ }^{22}$. Sa rencontre avec un fonds de manuscrits dont l'un, comme il ne pouvait l'ignorer ${ }^{23}$, était directement attaché à la transmission d'un document concernant Pascal laissait bien augurer du destin de l'Entretien. Mais la requête de Bonamy ne trouva finalement pas d'écho favorable auprès des supérieurs du Père Guerrier à l'Oratoire de Clermont. Le Père Guerrier ne vint à Paris que pour des séjours plus ou moins longs pour représenter sa maison aux assemblées générales de l'Oratoire. L' Entretien de Pascal avec M. de Sacy aurait trouvé en lui son éditeur idéal, l'ironique destin en avait décidé autrement.

$13 \mathrm{Au}$ reste, le jugement que l'oratorien portait sur les Mémoires de Nicolas Fontaine ne différait pas de beaucoup de celui de ses contemporains. Ainsi, note-t-il peu de temps après la parution des Mémoires, "J'ai lu depuis peu les Mémoires de M. Fontaine dont je n'ai pas été fort satisfait. J'y ai trouvé peu d'ordre, peu de faits intéressants, beaucoup de minuties, grand nombre de répétitions, et plusieurs choses qui ne m'ont point édifié... ", avant d'évoquer quelques passages des Mémoires où Fontaine, selon lui, développe des réflexions qui nuisent à l'image des Messieurs et de l'abbé de Saint-Cyran. Fontaine était loin de sortir du purgatoire où l'avaient paradoxalement placé les proches de Port-Royal.

Mais l'ironie du destin n'avait pas fini de déployer ses ruses. La bibliothèque de la Ville de Paris devint par la suite bibliothèque de l'Institut de France, et en 1928, son conservateur en chef Jean Tremblot de La Croix en publia le nouveau catalogue ${ }^{24}$. Dans un article consacré au parlementaire Antoine Moriau et à son père Guillaume, dont les collections composèrent le premier et inestimable noyau de la Bibliothèque de la Ville de Paris, Jean Tremblot de La Croix évoquait le sévère jugement des Mémoires secrets de Bachaumont ${ }^{25}:$ "Cet établissement au fond est mesquin, il coûte fort cher et ne contient rien de curieux", et commentait: "Quelle injustice à l'égard d'un fonds si précieux dans sa spécialisation et en même temps le seul à Paris qui ne fût pas encombré de théologie et de polémique janséniste $e^{26}$ !»

15 Gageons qu'il reste encore quelques chapitres à écrire au roman inachevé des Mémoires de Nicolas Fontaine. 


\section{NOTES}

1. M. Bouteron et J. Tremblot de La Croix, Catalogue général des manuscrits des bibliothèques de France, bibliothèque de l'Institut, ancien et nouveau fonds, Paris, Plon, 1928. Le manuscrit, en cinq volumes, est répertorié à la page 149 sous les cotes 664 à 668 .

2. Voir Pascal, Euvres complètes, t. III, éd. Jean Mesnard, Paris, Desclée de Brouwer, 1991, pp. 99-111, et Pascal, Entretien avec M. de Sacy, original inédit présenté par Pascale Mengotti et Jean Mesnard, Paris, Desclée de Brouwer, coll. «Les Carnets », 1994, pp. 40-45.

3. Voir Pascal, Entretien... éd. Les Carnets DDB, pp. 12-33

4. Nicolas Fontaine, Mémoires pour servir à l'histoire de Port-Royal, Utrecht, 1736, 2 vol.

5. Voir par exemple Jean Orcibal, Jean Duvergier de Hauranne abbé de Saint-Cyran et son temps (1581-1638), Les origines de Jansénisme III, Paris, Vrin, 1948, pp. 25-26.

6. Voir Jean Mesnard, « Le Maistre de Sacy et son secrétaire Fontaine », Chroniques de Port-Royal. Paris, $n^{\circ} 33,1984$, p. 5-18.

7. Lettre du 21 octobre 1731, citée par Sainte-Beuve, Port-Royal, Paris, Gallimard, «Bibliothèque de la Pléiade ", 1953, t. I, pp. 698-699.

8. Voir note 1.

9. Institut de France, ms 664, pages VII et VIII.

10. Il s'agit des propos de Sacy suivants : «Quelle nouvelle idée me donne-t-on de la grandeur de Dieu, disait-il, en me venant dire que le soleil est un amas de rognures, et que les bêtes sont des horloges ?» Et se riant doucement quand on lui parlait de ces choses, il témoignait plus plaindre ceux qui s'y arrêtaient, qu'avoir envie de s'y arrêter lui-même.

Il me dit un jour me parlant là-dessus en particulier, qu'il admirait la conduite de Dieu dans ces nouvelles opinions de M. Descartes où tout le monde entrait tête baissée, qu'il considérait dans M. Descartes et Aristote, comme un voleur qui venait tuer un autre voleur et lui enlever ses dépouilles ; qu'Aristote peu à peu était enfin devenu le maître des maîtres de l'Église. (Édition des Carnets DDB, pages 85-86.)

11. «J'ai vu en Sorbonne, me disait-il, et je ne le pus voir sans frémir, qu'un docteur citant un passage de l'Écriture, un autre le réfuta hardiment par un passage d'Aristote; ce qui étonna de telle sorte le premier qu'il répondit presque tout étourdi : "Valet hic scriptura sacra "; à quoi l'autre sans se démonter dit brusquement : «Valet et Aristoteles »; "Attamen, répliqua le premier, et scriptura sacra et Aristoteles. » Et après un si horrible brigandage il survient récemment un autre homme qui le pille et le tue. Tant mieux. Plus de morts moins d'ennemis. Il en arrivera peut-être autant ensuite à $\mathrm{M}$. Descartes. "

«Dieu a fait le monde pour deux choses, m'ajouta-t-il. L'une pour donner une grande idée de lui ; l'autre pour peindre les choses invisibles dans les visibles. M. Descartes détruit l'un et l'autre. Le soleil est un si bel ouvrage! lui dit-on. Point du tout, répond-il ; c'est un amas de rognures. Au lieu de reconnaître les choses invisibles dans les visibles, comme dans le soleil qui est le Dieu de la nature, et voir en tout ce qu'il produit dans les plantes l'image de la grâce, ils prétendent au contraire rendre raison de tout par de certains crochets qu'ils se sont imaginés. Je les compare à des ignorants qui verraient un admirable tableau et qui au lieu d'admirer l'ensemble, s'arrêteraient à chaque couleur en particulier et diraient : «Qu'est-ce que ce rouge-là ? De quoi est-il composé ? C'est de telle chose ; non c'est d'une autre "; au lieu de contempler tout le dessein du tableau dont la beauté charme les sages qui le considèrent.

«Je ne prétends pas, dit M. Descartes, dire les choses comme elles sont en effet. Le monde est un si grand objet qu'on s'y perd ; mais je le regarde comme un chiffre. Les uns tournent et retournent les lettres de cet alphabet, et trouvent quelque chose ; moi j'ai aussi trouvé quelque chose, mais ce n'est pas peut-être ce que Dieu a fait. » « Non, disait M. de Sacy, mais c'est, selon le 
langage des Pères : Infinita disputandi libido, tuncque demum magis ignorata veritas postquam praesumptum est quod posset agnosci. Ces gens-là cherchent la vérité à tâtons ; c'est un grand hasard quand ils la trouvent. Je les regarde comme je regardais l'enseigne du Cadran en passant sur le pont Notre-Dame. Le cadran disait vrai alors, et je disais : «Passons vite ; il n'y fera plus bon bientôt. C'est la vérité qui l'a rencontré, il n'a pas rencontré la vérité ; il ne dit vrai qu'une fois le jour. » (Édition des Carnets DDB, pages 86-88.)

12. Institut de France, ms. 664, page VII.

13. Institut de France, ms. 666, page 202 ; voir par ailleurs dans l'Entretien : « Cet esprit de paix de M. de Sacy l'avait aussi toujours porté à fuir toutes les disputes dans les sciences tant saintes que naturelles », éd. Carnets DDB, p. 83.

14. "Qu'on peut se tuer quand on est si persécuté qu'on doit croire que Dieu appelle, et à d'autres. » (ms. 666 page 229 ; éd. Carnets DDB p. 99).

15. Le correcteur préfère " pouvait soutenir une dispute avec lui » à " lui prêterait le collet » (ms. 666 page 226 ; éd. Carnets DDB p. 90.) ; « orgueil » à « superbe » (ms. 666, pages 229, 246 ; éd. Carnets DDB, pp. 98, 124), répondant ainsi à un désir d'atténuation ; « Ainsi nous qui avons la foi nous devons de même mettre à part tout ce qu'il dit » à " Ainsi nous qui avons la foi devons de même mettre à part tout ce qu'il dit » (ms. 666, page 239) ; " ...où s'accordent ces sentiments opposés incompatibles dans ces doctrines humaines. » corrige « ...où s'accordent ces opposés qui étaient incompatibles dans ces doctrines humaines " (ms. 666, page 248, éd. Carnets DDB p. 126), qui était la version authentique des propos de Pascal. Dans le reste de l'Entretien, les hardiesses du style de Pascal restent inaltérées.

16. P.N. Desmolets, Continuation des Mémoires de littérature et d'histoire, t. V, $2^{\mathrm{e}}$ partie, Paris, 1728 , pp. 239-270. Voir Pascal, Euvres Complètes, t. III, éd. Jean Mesnard, Paris, Desclée de Brouwer, 1991, pages 82, 94-99, l'étude de cette édition et de son rang dans la filiation de l'Entretien; ibidem, pp. 82, 88-90 l'étude de l'édition de Tronchay.

17. Franklin, Les anciennes bibliothèques de Paris, t. III, Paris, 1878, pp. 183-185.

18. Ibidem, pp. 211 ; délibération du 11 sept.1760, appendice IV, pp. 215-216.

19. BN, fds Joly de Fleury, ms 355, pièce 193, lettre du 19 juin 1760.

20. L'intention de disculper son ami de l'accusation de jansénisme actif paraîtra encore plus nettement en comparant ce portrait avec celui que fit l'abbé Micolon de Blanval dans son éloge funèbre du P. Pierre Guerrier, voir Pascal, O.C., éd. Jean Mesnard, t. I, pp. 1179-1183.

21. Voir Pascal, O.C., éd. Jean Mesnard, t. I, pp. 135-169.

22. Voir Pascal, O.C., éd. Jean Mesnard, t. I, pp. 139-141.

23. Pierre Guerrier ne pouvait ignorer l'origine de l'Entretien, grâce à Marguerite Perrier, qu'il fréquentait assidûment pendant la réalisation de ses recueils. La nièce de Pascal ignorait l'existence de cet écrit lorsque l'édition de Desmolets parut en 1728, et écrivit à son cousin l'abbé d'Etémare qui lui répondit dans une lettre datée du 20 juin 1728 que l'Entretien était tiré des Mémoires de Fontaine. Or la copie de cette lettre figure dans le Premier Recueil Guerrier, pp. 542-543. Voir Pascal, O.C., t. I, p. 318.Voir Pierre Courcelle, l'Entretien de Pascal et Sacy, ses sources et ses énigmes, Paris, Vrin, 1960, pp. 69-70, qui reproduit cette lettre.

24. Voir ci-dessus, note 1.

25. Bachaumont, Mémoires secrets, V, 1777, p. 212.

26. Jean Tremblot de La Croix, "Deux vrais bibliophiles ", Mélanges d'histoire littéraire et de bibliographie offerts à Jean Bonnerot, Paris, Nizet, 1954, p. 534. 
INDEX

Mots-clés : Pascal, Sacy, Fontaine (Nicolas), manuscrit, Entretien de Pascal avec M. de Sacy, édition

Keywords : Pascal, Sacy, Fontaine (Nicolas), manuscript, Entretien de Pascal avec M. de Sacy, edition 